\title{
Dynamics of quasi-isometric foliations
}

\author{
Andy Hammerlindl
}

September 18, 2018

\begin{abstract}
If the stable, center, and unstable foliations of a partially hyperbolic system are quasi-isometric, the system has Global Product Structure. This result also applies to Anosov systems and to other invariant splittings.

If a partially hyperbolic system on a manifold with an abelian fundamental group has quasi-isometric stable and unstable foliations, the center foliation is without holonomy. If, further, the system has Global Product Structure, then all center leaves are homeomorphic.
\end{abstract}

\section{Introduction}

Invariant foliations are invaluable in understanding the properties of smooth dynamical systems. In this paper, we examine foliations invariant under a dynamical system which have the additional property of quasi-isometry. A foliation $W$ is quasi-isometric if there is a constant $Q>1$ such that for any two points $x, y \in M$ which lie on the same leaf of $W, d_{W}(x, y) \leq Q d_{M}(x, y)+Q$ where $d_{W}$ is distance measured along the leaf and $d_{M}$ is distance measured on the manifold. Such foliations arise naturally in the study of certain partially hyperbolic systems. A diffeomorphism $f: M \rightarrow M$ on a compact Riemannian manifold is partially hyperbolic if there are a $T f$-invariant splitting, $T M=E^{s} \oplus E^{c} \oplus E^{u}$, an integer $n \geq 1$, and constants $\lambda<\hat{\gamma}<1<\gamma<\mu$ such that

$$
\left\|T f^{n} v^{s}\right\|<\lambda<\hat{\gamma}<\left\|T f^{n} v^{c}\right\|<\gamma<\mu<\left\|T f^{n} v^{u}\right\|
$$

for all $x \in M$, and unit vectors $v^{s} \in E^{s}(x), v^{c} \in E^{c}(x)$, and $v^{u} \in E^{u}(x)$.

For every partially hyperbolic system, there are unique foliations $W^{u}$ and $W^{s}$ tangent to the unstable $E^{u}$ and stable $E^{s}$ subbundles. In many but not all cases, there is also a foliation tangent to the center subbundle $E^{c}$. If the three subbundles $E^{c}, E^{c s}=E^{c} \oplus E^{s}$, and $E^{c u}=E^{c} \oplus E^{u}$ are uniquely integrable, the system is said to be dynamically coherent. M. Brin proved that if the foliations $W^{u}$ and $W^{s}$ are quasi-isometric when lifted to the universal cover of the manifold, the system is dynamically coherent 2. Later, M. Brin, D. Burago, and S. Ivanov proved that every partially hyperbolic system on the 3-torus is dynamically coherent by establishing this quasi-isometry property [4]. 
The property of quasi-isometry tells us much more about partially hyperbolic systems than just dynamical coherence. In my thesis, I used the property to give a classification result for partially hyperbolic systems on the 3 -torus [12].

This paper explores the consequences of quasi-isometry for partially hyperbolic systems on general manifolds and in any dimension $d \geq 3$. The first result is on the structure of the invariant foliations.

Theorem 1.1. Suppose $f: M \rightarrow M$ is partially hyperbolic and the foliations $W^{u}, W^{s}$, and $W^{c}$ are quasi-isometric when lifted to the universal cover, $\tilde{M}$. Then, the foliations $W^{c s}$ and $W^{c u}$ tangent to $E^{c} \oplus E^{s}$ and $E^{c} \oplus E^{u}$ are also quasi-isometric on $\tilde{M}$, and $f$ has Global Product Structure, that is, for every $x, y \in \tilde{M}:$

1. $W^{u}(x)$ and $W^{c s}(y)$ intersect exactly once,

2. $W^{s}(x)$ and $W^{c u}(y)$ intersect exactly once,

3. if $x \in W^{c s}(y)$, then $W^{c}(x)$ and $W^{s}(y)$ intersect exactly once, and

4. if $x \in W^{c u}(y)$, then $W^{c}(x)$ and $W^{u}(y)$ intersect exactly once.

In the classification given for systems on the 3-torus, the first key step of the proof was establishing Global Product Structure. As such, Theorem 1.1 may help to classify systems on other manifolds and in higher dimensions. Section 2 gives specific examples of its application and the proof of the theorem is given in Section 3 .

There are partially hyperbolic systems where $W^{u}$ and $W^{s}$ are quasi-isometric, but $W^{c}, W^{c s}$, and $W^{c u}$ are not [11. These examples still have Global Product Structure.

Question 1.2. If $f: M \rightarrow M$ is partially hyperbolic, and the foliations $W^{u}$ and $W^{s}$ (but not necessarily $W^{c}$ ) are quasi-isometric on the universal cover, does $f$ have Global Product Structure?

On the 3-torus, partially hyperbolic systems fall into two categories: skew products, where every center leaf is a compact circle, and "Derived-from-Anosov" systems, where the center foliation consists entirely of lines. In either case, any two leaves of the center foliation are homeomorphic, and this is indicative of a more general principle.

Theorem 1.3. Suppose $M$ is a compact manifold with an abelian fundamental group, $f: M \rightarrow M$ is partially hyperbolic with Global Product Structure, and $W^{s}$ and $W^{u}$ are quasi-isometric on the universal cover. Then, any two leaves of $W^{c}$ are homeomorphic.

Corollary 1.4. Suppose $M$ is a compact manifold with an abelian fundamental group, $f: M \rightarrow M$ is partially hyperbolic and $W^{u}, W^{c}$, and $W^{s}$ are quasiisometric on the universal cover. Then, any two leaves of $W^{c}$ are homeomorphic. 
The assumption of an abelian fundamental group cannot be removed, as shown by the following example. Let $M=\mathbb{T}^{2} \times \mathbb{S}^{2}$ and let $A: \mathbb{T}^{2} \rightarrow \mathbb{T}^{2}$ be a hyperbolic toral automorphism so that $f=A \times$ id $: M \rightarrow M$ is partially hyperbolic and each center leaf is a sphere $\{x\} \times \mathbb{S}^{2}$. If $-y$ denotes the antipode of $y \in \mathbb{S}^{2}$, then $f$ commutes with $\tau: M \rightarrow M,(x, y) \mapsto(-x,-y)$ and the resulting diffeomorphism on the quotient space $M / \tau$ has four leaves homeomorphic to the projective plane while the rest of the leaves are spheres.

Question 1.5. If $f: M \rightarrow M$ is partially hyperbolic and $W^{u}$ and $W^{s}$ are quasi-isometric when lifted to the universal cover, then is there a finite cover of $M$ on which any two leaves of $W^{c}$ homeomorphic?

The key to proving Theorem 1.3 is to consider holonomies along the center foliation. We say that $W^{c}$ is without holonomy if the holonomy along every closed loop on a leaf of $W^{c}$ is the identity. Using this, Theorem 1.3 can be decomposed into two results, each interesting in its own right.

Theorem 1.6. Suppose $M$ is a compact connected manifold with an abelian fundamental group, and $f: M \rightarrow M$ is partially hyperbolic. If $W^{u}$ and $W^{s}$ are quasi-isometric when lifted to the universal cover, then the foliations $W^{c}, W^{c s}$, and $W^{c u}$ are without holonomy.

Theorem 1.7. Suppose $M$ is a connected manifold and $f: M \rightarrow M$ is partially hyperbolic. If the center foliation $W^{c}$ exists and is without holonomy, and $f$ has Global Product Structure, then all leaves of $W^{c}$ (on $M$ ) are homeomorphic.

The proof of Theorem [1.6 is based on properties of liftings of freely homotopic curves. These properties rely on the fundamental group being abelian and do not hold even for the most obvious generalization to manifolds with nilpotent fundamental groups. That said, the only counterexamples we know of are systems such as the example given above, where the center foliation lifts to a trivial fibering on a finite cover.

Question 1.8. Suppose $M$ is a compact connected manifold, $f: M \rightarrow M$ is partially hyperbolic, and $W^{u}$ and $W^{s}$ are quasi-isometric when lifted to the universal cover. Modulo a lift to a finite cover, is the center foliation $W^{c}$ without holonomy?

The author's aim in researching this topic was to determine exactly which 3dimensional manifolds support partially hyperbolic systems with quasi-isometric invariant foliations. Alas, this goal has remained elusive.

Question 1.9. For which manifolds $M$ is there $f: M \rightarrow M$ is partially hyperbolic, with $\operatorname{dim} E^{s}=\operatorname{dim} E^{c}=\operatorname{dim} E^{u}=1$, and $W^{u}$ and $W^{s}$ quasi-isometric on the universal cover?

The following fact shows that positive answers to Questions 1.2 and 1.8 will give an answer to Question 1.9 . 
Theorem 1.10. If $f: M \rightarrow M$ is partially hyperbolic with Global Product Structure, $\operatorname{dim} E^{s}=\operatorname{dim} E^{c}=\operatorname{dim} E^{u}=1$, and $W^{c s}$ (or $W^{c u}$ ) is without holonomy, then $M$ a circle bundle over $\mathbb{T}^{2}$.

Section 4 contains the proofs of Theorems 1.6, 1.7, and 1.10,

Above, we stated Theorem 1.1 in terms of the three-way splitting of a partially hyperbolic system. In fact, the theorem generalizes to arbitrary $n$-way splittings. As this requires more notation to state, we leave the exposition until Section 3. In an Anosov system, $T M$ has a two-way splitting into contracting $E^{s}$ and expanding $E^{u}$ bundles, and the result can be stated as follows.

Theorem 1.11. If $f: M \rightarrow M$ is Anosov and $W^{u}$ and $W^{s}$ are quasi-isometric on the universal cover $\tilde{M}$, then $f$ has Global Product Structure, that is, for every $x, y \in \tilde{M}$, the intersection $W^{s}(x) \cap W^{u}(y)$ consists of exactly one point.

If $f: M \rightarrow M$ is an Anosov diffeomorphism, it is a famous open question if $M$ is finitely covered by a nilmanifold. Further, it is not known if the non-wandering set of $f$ is equal to $M$ or if the universal cover of $M$ is homeomorphic to $\mathbb{R}^{n}$. In 1, Brin defined pinching conditions on the derivative of an Anosov system under which the system necessarily has Global Product Structure (referred to in that paper as the property of "infinite extension"). He further showed that if an Anosov diffeomorphism has Global Product Structure, then its non-wandering set is the entire manifold, and the universal cover is homeomorphic to $\mathbb{R}^{n}$. As such, Theorem 1.11 shows that any Anosov diffeomorphism with quasi-isometric foliations also enjoys these properties.

Corollary 1.12. If $f: \underset{\sim}{M} \rightarrow M$ is Anosov and $W_{\tilde{M}}^{u}$ and $W^{s}$ are quasi-isometric on the universal cover $\tilde{M}$, then $\Omega(f)=M$ and $\tilde{M}$ is homeomorphic to $\mathbb{R}^{n}$.

One can find hyperbolic toral automorphisms which are not pinched, but as the invariant foliations are linear, they are quasi-isometric when lifted to the universal cover. The work of J. Franks and A. Manning shows that every Anosov diffeomorphism on a torus must have quasi-isometric lifted foliations [9] 15. In Section 5, we examine a six-dimensional nilmanifold supporting Anosov diffeomorphisms, some of which have quasi-isometric lifted foliations while others do not.

\section{Applications}

Attempts to classify partially hyperbolic diffeomorphisms have proved frustrating. We do not know if examples coming from algebraic maps and time-one maps of flows, or from perturbations of these diffeomorphisms are indicative of the general behaviour of partially hyperbolic systems.

One special successful case is for 3-manifolds with nilpotent fundamental groups. In this setting, the only manifolds supporting partially hyperbolic diffeomorphisms are 3-dimensional nilmanifolds, that is, circle bundles over $\mathbb{T}^{2}[16$. These are the only manifolds where all partially hyperbolic systems have been 
classified up to leaf conjugacy, a notion analogous to topological equivalence for flows [12]. 11 .

These manifolds are also the only known manifolds for which all partially hyperbolic systems have stable and unstable foliations which are quasi-isometric when lifted to the universal cover [16]. While this could be demonstrated as a consequence of the classification, such reasoning would be circular. In fact, the proof of classification starts by establishing geometric properties of the foliation, including that of quasi-isometry; using these properties to establish Global Product Structure; then using the Global Product Structure to construct the leaf conjugacy. The proof relies on results specific to the 3-dimensional manifolds 6 . While there are more difficulties in higher dimensions, the same general approach might work, in which case, Theorem 1.1 would be a critical step in the reasoning.

As a simple example of where Theorem 1.1 applies, consider affine maps of the form

$$
\mathbb{R}^{d} \rightarrow \mathbb{R}^{d}, \quad x \mapsto A x+b
$$

where $A \in G L(n, \mathbb{Z})$ and $b \in \mathbb{R}^{d}$. If such a map has a partially hyperbolic splitting, the resulting leaves of the invariant foliations are translates of subspaces of $\mathbb{R}^{d}$ and so the foliations are quasi-isometric.

In 2, Proposition 5], Brin considers a specific example of an affine map which quotients down to a partially hyperbolic diffeomorphism $f_{0}: \mathbb{T}^{3} \rightarrow \mathbb{T}^{3}$. He shows that every $f$ which is $\epsilon-C^{1}$-close to $f_{0}$ is partially hyperbolic and has quasi-isometric invariant foliations. Further, he gives an actual value for the size of this neighbourhood: $\epsilon=0.1$.

This technique readily extends to any affine map $g_{0}: \mathbb{T}^{d} \rightarrow \mathbb{T}^{d}$. Given the eigenvalues associated to $g_{0}$, we may calculate a value for $\epsilon$ such that every $g$ $\epsilon-C^{1}$-close has quasi-isometric invariant foliations. Then, Theorem 1.1 tells us that every system in this macroscopic open set around $g_{0}$ has Global Product Structure.

Suppose further that $g_{0}$ is a skew-product, that is, its center leaves are compact. Take a subset $U \subset \mathbb{T}^{d}$ and a diffeomorphism $h: \mathbb{T}^{d} \rightarrow \mathbb{T}^{d}$ whose support lies in $U$. If $U$ is disjoint from the orbit of a periodic center leaf of $g_{0}$, then $g:=h \circ g_{0}$ will preserve this leaf. If $h$ is sufficently $C^{1}$-close to the identity, then, as above, $W^{s}$ and $W^{u}$ are quasi-isometric, and by Theorem [1.6] every leaf is compact and the center foliation is a trivial fiber bundle. Even in cases where $h$ is large in the $C^{1}$ norm, we may still be able to establish that $W^{s}$ and $W^{u}$ are quasi-isometric (say, by [2, Proposition 4]). Then, the same consequences follow. These techniques apply, with minor adaptations, to more general skew products on manifolds of the form $\mathbb{T}^{d} \times N$ where $N$ has an abelian fundamental group.

\section{Splittings}

We now state and prove several results for invariant splittings, from which Theorem 1.1 follows as a consequence. 
Throughout this section, assume $M$ is a Riemannian manifold (not necessary compact or connected, but without boundary) and that $f: M \rightarrow M$ is a $C^{1}$ diffeomorphism. Also assume that the derivative $T f$ is uniformly bounded on $M$, which is always the case when $M$ is compact or when $f$ is lift of a map on a compact manifold to the universal cover.

Definition. A $T f$-invariant subbundle $E \subset T M$ is contracting if there is $n>0$ such that $\left\|T f^{n} v\right\|<\frac{1}{2}$ for every unit vector $v \in E$. A $T f$-invariant subbundle $E \subset T M$ is expanding if there is $n>0$ such that $\left\|T f^{n} v\right\|>2$ for every unit vector $v \in E$.

Remark. Note that by this definition the zero bundle $0 \subset T M$ is both contracting and expanding. It is the only subbundle to satisfy both properties.

Definition. Suppose $E^{1}, E^{2}$ are continuous $T f$-invariant subbundles of $T M$. We say that $E^{2}$ dominates $E^{1}$ if there is $n>0$ such that

$$
\left\|T f^{n} u_{x}\right\|<\frac{1}{2}\left\|T f^{n} v_{x}\right\|
$$

for all $x \in M$ and unit vectors $u_{x} \in E_{x}^{1}, v_{x} \in E_{x}^{2}$. Further, $E^{2}$ absolutely dominates $E^{1}$ if there is $n>0$ such that

$$
\left\|T f^{n} u_{x}\right\|<\frac{1}{2}\left\|T f^{n} v_{y}\right\|
$$

for all $x, y \in M$ and unit vectors $u_{x} \in E_{x}^{1}, v_{y} \in E_{y}^{2}$

Remark. If $E^{2}$ dominates $E^{1}$, it is clear from the definition that $E^{1} \cap E^{2}=0$.

Remark. Absolute domination is stronger just domination as it compares the effect of the derivative at different points. An equivalent definition of absolute domination is that there are constants $0<\gamma<\mu$ and an integer $n>0$ such that

$$
\left\|T f^{n} u_{x}\right\|<\gamma<\mu<\left\|T f^{n} v_{x}\right\|
$$

for all $x \in M$ and unit vectors $u_{x} \in E_{x}^{1}, v_{x} \in E_{x}^{2}$. From this, one can show that at least one of $E^{1}$ and $E^{2}$ is either expanding or contracting.

Definition. A splitting of a continuous $T f$-invariant subbundle $E \subset T M$ is a sum of the form

$$
E=E^{1} \oplus \cdots \oplus E^{n}
$$

where each $E^{i}$ is continuous and $T f$-invariant. A splitting is (absolutely) dominated if the subbundles may be ordered such that $E^{j}$ (absolutely) dominates $E^{i}$ for $1 \leq i<j \leq n$.

Remark. Extending the previous remark, one sees that in an absolutely dominated splitting, there can be at most one subbundle $E^{i}$ which is neither expanding nor contracting.

Remark. To make explicit the order of the subbundles in the dominated splitting, we sometimes write

$$
E=E^{1} \oplus_{<} E^{2} \oplus_{<} \cdots \oplus_{<} E^{n}
$$

where $E^{i} \oplus_{<} E^{j}$ signifies that $E^{j}$ dominates $E^{i}$. 
With this notation, we can now give succinct definitions for notions introduced in the previous section.

Definition. The diffeomorphism $f: M \rightarrow M$ is Anosov if there is a splitting $T M=E^{s} \oplus E^{u}$ such that $E^{s}$ is contracting and $E^{u}$ is expanding.

Definition. The diffeomorphism $f: M \rightarrow M$ is partially hyperbolic if there is an absolutely dominated splitting $T M=E^{s} \oplus_{<} E^{c} \oplus_{<} E^{u}$ such that $E^{s}$ is contracting, $E^{u}$ is expanding, and $0 \neq E^{c} \neq T M$.

If both $E^{s}$ and $E^{u}$ are non-zero then $f$ is partially hyperbolic in the strong sense. Otherwise, it is partially hyperbolic in the weak sense.

Remark. This definition of partial hyperbolicity is sometimes called absolute partial hyperbolicity, in contrast to point-wise partial hyperbolicity, where the dominated splitting $T M=E^{s} \oplus_{<} E^{c} \oplus_{<} E^{u}$ need not be an absolutely dominated splitting. In this more general setting, F. Rodriguez-Hertz, M. A. RodriguezHertz, and R. Ures have announced a non-dynamically coherent example in $\mathbb{T}^{3}$. This example suggests that quasi-isometry is useful only in studying the absolute case. Indeed, all of the proofs in this paper rely on the absolute version of the definition.

Definition. A subbundle $E \subset T M$ is integrable if there is a foliation $\mathcal{F}$ such that $T \mathcal{F}=E$. It is uniquely integrable if every curve $\gamma:[0,1] \rightarrow M$ tangent to $E$ lies in a single leaf of $\mathcal{F}$.

Theorem 3.1 (Brin-Pesin [5], Hirsch-Pugh-Shub [13]). If $f$ is partially hyperbolic, then $W^{u}$ and $W^{s}$ are uniquely integrable.

To prove Theorem 3.1 one constructs the foliations by a graph transformation argument. The same argument applies when restricted to a leaf of an invariant foliation, and so can be generalized.

Proposition 3.2. If $f$ has a $T f$-invariant, uniquely integrable subbundle $E \subset$ $T M$ with dominated splitting $E=E^{c} \oplus_{<} E^{u}$ and $E^{u}$ is expanding, then $E^{u}$ is uniquely integrable.

Let $d=d_{M}$ denote distance measured on the manifold $M$.

Definition. A foliation $W$ on $M$ is quasi-isometric if there is $Q>1$ such that $d_{W}(x, y) \leq Q d(x, y)$ for any $x, y \in M$ which lie on the same leaf of $W$.

The standard definition of quasi-isometry would use an inequality of the form

$$
d_{W}(x, y) \leq Q d(x, y)+Q .
$$

In this paper, we only consider foliations with $C^{1}$-leaves which are tangent to a uniformly continuous subbundle $E \subset M$. In this case, one can show that as $d(x, y) \rightarrow 0$, the ratio between $d_{W}(x, y)$ and $d(x, y)$ tends uniformly to one. Therefore (up to a change of the value $Q$ ) the two definitions are equivalent.

As stated in the introduction, quasi-isometry implies dynamical coherence.

Theorem 3.3 (Brin [2]). If $f$ is partially hyperbolic, and $W^{u}$ and $W^{s}$ are quasiisometric when lifted to the universal cover, then $E^{c}, E^{c s}$, and $E^{c u}$ are uniquely integrable. 
Note that Theorems 3.1 and 3.3 hold for partial hyperbolicity in the weak or strong sense. If $E^{s}$ or $E^{u}$ is equal to the zero bundle, it is uniquely integrable and the tangent foliation, where each leaf consists of a single point, is trivially quasi-isometric. Theorem 3.3 can be formulated with respect to an invariant foliation.

Proposition 3.4. If $f$ has a $T f$-invariant, uniquely integrable subbundle $E \subset$ $T M$ with absolutely dominated splitting $E=E^{c} \oplus<E^{u}$ and $E^{u}$ is expanding and tangent to a quasi-isometric foliation, then $E^{c}$ is uniquely integrable.

We now build up the knowledge needed to prove Theorem 1.1 starting with a technical lemma.

Lemma 3.5. Suppose $f$ has a continuous, $T f$-invariant subbundle $E \subset T M$ with absolutely dominated splitting $E=E^{c} \oplus_{<} E^{u}$ and that $E^{c}$ and $E^{u}$ are tangent to quasi-isometric foliations $W^{c}$ and $W^{u}$. Then, there is $c>0$ such that

$$
\max \{d(x, y), d(x, z)\} \leq c d(y, z)
$$

for all $x \in M, y \in W^{u}(x)$, and $z \in W^{c}(x)$.

Remark. Since the foliations are quasi-isometric, we may, with at most a change in the constant $c$, conclude further that

$$
\max \left\{d_{u}(x, y), d_{c}(x, z)\right\} \leq c d(y, z)
$$

where $d_{u}$ and $d_{c}$ are the distances measured along the foliations.

Remark. In this lemma, we need not assume that $E$ is integrable.

Proof. Without loss of generality, by replacing $f$ by an iterate $f^{n}$, there are constants $\gamma<\mu$ such that $\|T f v\| \leq \gamma\|v\|$ for $v \in E^{c}$, and $\mu\|v\| \leq\|T f v\|$ for $v \in E^{u}$. By standing assumption, the derivative $T f$ is bounded; that is, there is $\lambda>0$ such that $\|T f v\| \leq\|v\|$ for all $v \in T M$.

Let $x, y, z \in M$ be as in the hypothesis.

Case One: Suppose $2 d(x, y) \leq d(x, z)$. Then, using the triangle inequality,

$$
d(x, z)=2 d(x, z)-d(x, z) \leq 2 d(x, z)-2 d(x, y) \leq 2 d(y, z) .
$$

Thus, the theorem holds in this case with the caveat that $c \geq 2$.

Case Two: Suppose $d(x, z)<2 d(x, y)$. Let $Q>1$ be a constant of quasiisometry satisfied by both foliations. Then

$$
d\left(f^{n}(x), f^{n}(z)\right) \leq d_{c}\left(f^{n}(x), f^{n}(z)\right) \leq \gamma^{n} d_{c}(x, z) \leq Q \gamma^{n} d(x, z)
$$

and

$$
Q d\left(f^{n}(x), f^{n}(y)\right) \geq d_{u}\left(f^{n}(x), f^{n}(y)\right) \geq \mu^{n} d_{u}(x, y) \geq \mu^{n} d(x, y) .
$$


These estimates combine to give

$$
\begin{aligned}
d\left(f^{n}(x), f^{n}(y)\right) & \leq d\left(f^{n}(x), f^{n}(z)\right)+d\left(f^{n}(y), f^{n}(z)\right) \quad \Rightarrow \\
Q^{-1} \mu^{n} d(x, y) & \leq Q \gamma^{n} d(x, z)+\lambda^{n} d(y, z) \quad \Rightarrow \\
Q^{-1} \mu^{n} d(x, y) & \leq 2 Q \gamma^{n} d(x, y)+\lambda^{n} d(y, z) \quad \Rightarrow \\
\left(Q^{-1} \mu^{n}-2 Q \gamma^{n}\right) d(x, y) & \leq \lambda^{n} d(y, z) .
\end{aligned}
$$

Fixing $n$ large enough that $Q^{-1} \mu^{n}-2 Q \gamma^{n}>0$, the last line above simplifies to $d(x, y) \leq c_{0} d(y, z)$ for an appropriate positive constant $c_{0}$ independent of $x, y$, and $z$. As we are considering the case $d(x, z)<2 d(x, y)$, it follows that

$$
\max \{d(x, y), d(x, z)\} \leq 2 c_{0} d(y, z) .
$$

Taking $c=\max \left\{2,2 c_{0}\right\}$ establishes the desired inequality for Cases One and Two and concludes the proof.

Theorem 3.6. Suppose $f$ has a continuous, $T f$-invariant subbundle $E \subset T M$ which is uniquely integrable with tangent foliation $W$. Suppose further that $E$ has an absolutely dominated splitting $E=E^{c} \oplus_{<} E^{u}$ and $W^{c}$ and $W^{u}$ are quasiisometric foliations tangent to $E^{c}$ and $E^{u}$. Then, for any two points $x$ and $y$ on a leaf of $W$, the intersection $W^{c}(x) \cap W^{u}(y)$ consists of exactly one point.

Proof. First, consider the case where $E=T M$ and the leaves of $W$ are the connected components of $M$.

To prove uniqueness, assume $x$ and $y$ lie on the same $W^{c}$ leaf and the same $W^{u}$ leaf. Lemma 3.5 with $y=z$ states that

$$
\max \{d(x, y), d(x, y)\} \leq c d(y, y)=0,
$$

which is a convoluted way of saying $x=y$.

To establish existence of the intersection, first fix a leaf $L$ of $W^{c}$. Define, for $T>0$,

$$
A_{T}=\bigcup_{x \in L} A_{T}^{u}(x) \quad \text { where } \quad A_{T}^{u}(x)=\left\{y \in W^{u}(x): d_{u}(x, y) \leq T\right\}
$$

and

$$
B_{T}=\bigcup_{x \in L} B_{T}^{u}(x) \quad \text { where } \quad B_{T}^{u}(x)=\left\{y \in W^{u}(x): d_{u}(x, y)=T\right\} .
$$

As the leaf $L$ is transverse to $W^{u}$, one can show by continuity of the foliation that the $d_{u}$-distance from a point $y \in A_{T}$ to $L$ depends continuously on $y$. Therefore, if $y \in A_{T}^{u}(x)$ and $d_{u}(x, y)<T$ then $y$ is in the interior of $A_{T}$, and consequently $\partial A_{T} \subset B_{T}$.

For some value $T>0$, suppose $x \in L$ and $y \in B_{T}^{u}(x)$. Note that the leaf $L$ is properly embedded in $M$, for if it accumulated on a point $p$, then $W^{u}(p)$ would intersect $L$ more than once. Then, the intersection of $L$ with a large closed ball 
centered at $y$ is a compact set. The function $z \mapsto d(y, z)$ defined on this compact set achieves its minimum at a point $z \in L$ such that $\operatorname{dist}(y, L)=d(y, z)$. The points $x, y, z$ satisfy the conditions of Lemma 3.5] and there is a constant $c>0$ (independent of $T)$ such that $d_{u}(x, y) \leq c d(y, z)$. Then,

$$
\operatorname{dist}(y, L)=d(y, z) \geq \frac{1}{c} d_{u}(x, y)=\frac{T}{c} .
$$

As this holds for all $y \in \partial A_{T} \subset B_{T}$, it follows that $\operatorname{dist}\left(\partial A_{T}, L\right) \geq \frac{T}{C}$. Any $y$ in the same component as $x$ is a finite distance away from $L$. Then, $y$ is in $A_{T}$ for sufficiently large $T$ and hence $W^{u}(y)$ intersects $L$.

In the case where $E \neq T M$, consider the manifold $\mathfrak{L}$ defined as the disjoint union of the leaves of the foliation $W$. There is a canonical bijective map $i: \mathfrak{L} \rightarrow M$, so we may define a Riemannian metric on $\mathfrak{L}$ by declaring $i$ on each leaf to be an isometry to its image. Further, there is a unique diffeomorphism $g: \mathfrak{L} \rightarrow \mathfrak{L}$ such that $i \circ g=f \circ i$. The proof reduces to the previous case by considering $g, \mathfrak{L}$, and $E=T \mathfrak{L}$ in place of $f, M$, and $E=T M$.

Theorem 3.7. Suppose $f$ has a continuous, $T f$-invariant, uniquely integrable subbundle $E \subset T M$ with absolutely dominated splitting

$$
E=E^{1} \oplus<E^{2} \oplus<\cdots \oplus<E^{n}
$$

such that each $E^{i}$ is tangent to a quasi-isometric foliation. Then each subbundle of the form $E^{j} \oplus \cdots \oplus E^{k}$ for $1 \leq j \leq k \leq n$ is uniquely integrable and the tangent foliation is quasi-isometric.

To prove this theorem, first consider the case $n=2$.

Lemma 3.8. Suppose $f$ has a continuous, $T f$-invariant, uniquely integrable subbundle $E \subset T M$ with absolutely dominated splitting $E=E^{c} \oplus<E^{u}$ such that $E^{c}$ and $E^{u}$ are tangent to quasi-isometric foliations $W^{c}$ and $W^{u}$. Then, the foliation tangent to $E$ is quasi-isometric.

Proof. Suppose $\Phi(x, y, z)$ and $\Psi(x, y, z)$ are real-valued formulae which are welldefined when $x \in M, y \in W^{u}(x)$, and $z \in W^{c}(x)$. Adopt the notation $\Phi(x, y, z) \asymp \Psi(x, y, z)$ if there is a constant $K>1$ such that

$$
\frac{1}{K} \Psi(x, y, z) \leq \Phi(x, y, z) \leq K \Psi(x, y, z)
$$

for all such triples of points.

For instance, with this notation, Lemma 3.5 can be concisely stated as

$$
\max \left\{d_{M}(x, y), d_{M}(x, z)\right\} \asymp d_{M}(y, z)
$$

where $d=d_{M}$ is the metric on $M$. Here, we have applied the lemma to the diffeomorphism $f$ on the manifold $M$. As in the proof of Theorem 3.6. let $\mathfrak{L}$ be the disjoint union of the leaves of $W$ with $i: \mathfrak{L} \rightarrow M$ the canonical bijection 
and $g: \mathfrak{L} \rightarrow \mathfrak{L}$ defined by the relation $i \circ g=f \circ i$. Applying Lemma 3.5 to $g$ implies that

$$
\max \left\{d_{W}(x, y), d_{W}(x, z)\right\} \asymp d_{W}(y, z)
$$

where $d_{W}$ denotes the distance along a leaf of $W$.

Using the quasi-isometry of $W^{u}$ in $M$,

$$
d_{M}(x, y) \leq d_{W}(x, y) \leq d_{u}(x, y) \leq Q d_{M}(x, y)
$$

so that $d_{W}(x, y) \asymp d_{M}(x, y)$ and similarly $d_{W}(x, z) \asymp d_{M}(x, z)$. These two relations combine to give

$$
\max \left\{d_{W}(x, y), d_{W}(x, z)\right\} \asymp \max \left\{d_{M}(x, y), d_{M}(x, z)\right\} .
$$

It is clear that the relation " $\asymp$ " is transitive, so that the relations (1), (2), and (3) imply

$$
d_{W}(y, z) \asymp d_{M}(y, z) .
$$

Now, if $y$ and $z$ are any two points on the same leaf of $W$, Theorem 3.6 implies that there is $x \in W^{u}(y) \cap W^{c}(z)$. That is, $(x, y, z)$ is a valid triple and the above relation (4) states that the distances $d_{W}(y, z)$ and $d_{M}(y, z)$ differ by at most a bounded proportion; in other words, the foliation $W$ is quasi-isometric.

Proof of Theorem 3.7. Assume that the theorem has already been proven for all splittings with less than $n$ summands and consider a splitting with exactly $n$ summands. Define $E(i, j)=E^{i} \oplus \cdots \oplus E^{j}$, so that $E=E(1, n)$ is the full bundle. For each $i, E=E(1, i) \oplus_{<} E(i+1, n)$ is an absolutely dominated splitting and either $E(1, i)$ is contracting or $E(i+1, n)$ is expanding. Assume the latter holds. Then, Proposition 3.2 implies that $E(i+1, n)$ is uniquely integrable. By the inductive hypothesis, all of the subbundles $E(j, k)$ for $i+1 \leq j \leq k \leq n$ are tangent to quasi-isometric foliations. In particular, the foliation tangent to $E(i+1, n)$ is quasi-isometric and Proposition 3.4 implies $E(1, i)$ is uniquely integrable.

We have shown that every bundle of the form $E(1, i)$ or $E(j, n)$ is uniquely integrable. As the property of unique integrability survives under intersection, each $E(i, j)=E(1, j) \cap E(i, n)$ is uniquely integrable. The resulting foliation tangent to $E(i, j)$ is quasi-isometric when $j-i<n-1$ due to the inductive hypothesis.

To see that the foliation tangent to $E=E(1, n)$ is quasi-isometric, write it as $E(1,1) \oplus E(2, n)$ and apply Lemma 3.8 .

Theorem 1.1 now follows as a consequence of Theorems 3.6 and 3.7

\section{Holonomy}

Definition. Let $\mathcal{F}$ be a foliation on a manifold $M, \alpha$ a closed curve on a leaf of $\mathcal{F}$, and $\tau \subset M$ a small plaque transverse to $\mathcal{F}$ passing through a point $x$ on $\alpha$ and such that $\operatorname{dim} \mathcal{F}+\operatorname{dim} \tau=\operatorname{dim} M$. Then, there is a small neighbourhood 


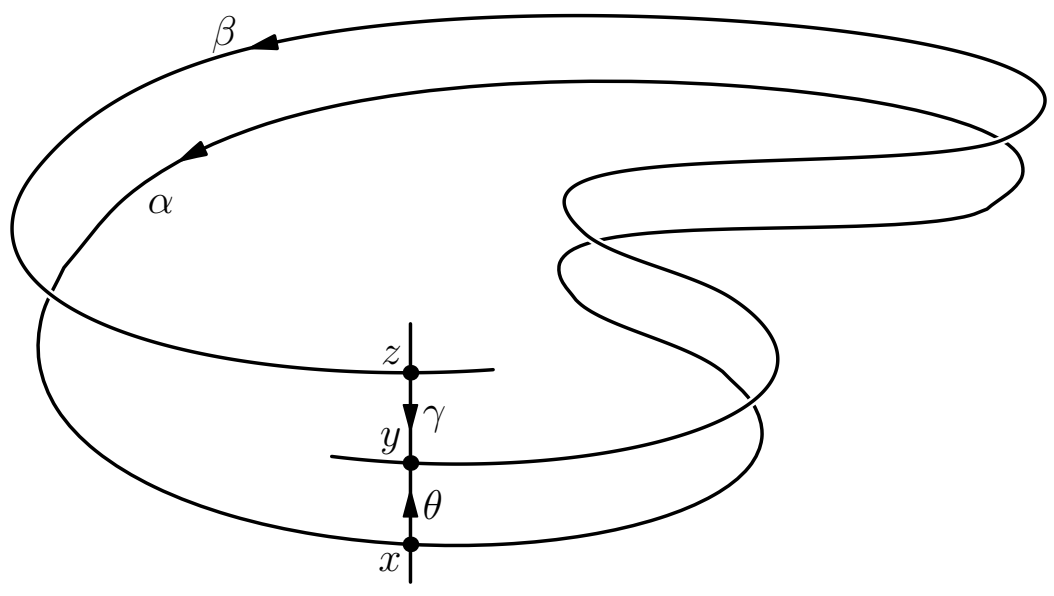

Figure 1: A non-trivial holonomy.

$\tau_{0} \subset \tau$ containing $x$ and a map $h: \tau_{0} \rightarrow \tau$ defined by following leaves of $\mathcal{F}$ along paths close to $\alpha$. The germ of this map is the holonomy along $\alpha$. If, for a foliation $\mathcal{F}$, every such choice of $\alpha, \tau$, and $\tau_{0}$ yields a map $h: \tau_{0} \rightarrow \tau$ which is the identity map, we say that $\mathcal{F}$ is without holonomy.

Proposition 4.1. Suppose $M$ is a compact connected manifold with an abelian fundamental group, and $f: M \rightarrow M$ is partially hyperbolic. If $W^{u}$ and $W^{s}$ are quasi-isometric when lifted to the universal cover, then $W^{c}$ is without holonomy.

Remark. This is a restatement of Theorem 1.6. The two are equivalent as the center-stable bundle of a diffeomorphism which is partially hyperbolic in the strong sense can always be viewed as the center bundle of the same system regarded as partially hyperbolic in the weak sense.

Proof. First, consider the weak sense of partial hyperbolicity where $E^{s}=0$.

Suppose holonomy along the center foliation is non-trivial along a closed path $\alpha:[0,1] \rightarrow W^{c}(x)$ based at a point $x \in M$. We are free to consider the holonomy as defined on a small plaque of $W^{u}(x)$. Then, as depicted in Figure [1. there are are distinct points $y, z \in W^{u}(x)$ and paths $\beta:[0,1] \rightarrow W^{c}(y)$, $\gamma, \theta:[0,1] \rightarrow W^{u}(x)$ such that

$$
\begin{array}{ll}
\alpha(0)=x & \alpha(1)=x \\
\beta(0)=y & \beta(1)=z \\
\gamma(0)=z & \gamma(1)=y \\
\theta(0)=x & \theta(1)=y
\end{array}
$$

and the concatenation $\theta \cdot \beta \cdot \gamma \cdot \theta^{-1}$ is homotopic to $\alpha$. For $n \geq 0$, define

$$
x_{n}=f^{n}(x), \quad y_{n}=f^{n}(y), \quad \alpha_{n}=f^{n} \circ \alpha, \quad \beta_{n}=f^{n} \circ \beta, \quad \gamma_{n}=f^{n} \circ \gamma .
$$


Define paths $\phi_{n}:[0,1] \rightarrow M$, such that $\phi_{n}(0)=x_{n}, \phi_{n}(1)=y_{n}$ and

$$
\text { length } \phi_{n} \leq \operatorname{diam} M \text {. }
$$

Note that, unlike the above sequences, we do not require $\phi_{n}$ to be equal to $f^{n} \circ \phi_{0}$. Also note that

$$
\left[\alpha_{n}\right]=\left[\theta_{n} \cdot \beta_{n} \cdot \gamma_{n} \cdot \theta_{n}^{-1}\right]=\left[\phi_{n} \cdot \beta_{n} \cdot \gamma_{n} \cdot \phi_{n}^{-1}\right] \in \pi_{1}\left(M, x_{n}\right)
$$

where last equality follows by conjugating by $\left[\phi_{n} \cdot \theta_{n}^{-1}\right]$ and using the assumption that the fundamental group is abelian.

Now consider the universal cover $\tilde{M}$. Choose $\tilde{x}_{n} \in \tilde{M}$ over each point $x_{n}$ and lift each curve $\alpha_{n}$ to a curve $\tilde{\alpha}_{n}$ whose starting point $\tilde{\alpha}_{n}(0)$ is $\tilde{x}_{n}$.

Lift $\phi_{n}, \beta_{n}, \gamma_{n}$, and again $\phi_{n}$ to curves $\tilde{\phi}_{n}, \tilde{\beta}_{n}, \tilde{\gamma}_{n}$, and $\tilde{\tilde{\phi}}_{n}$ so that the concatenation $\tilde{\omega}_{n}:=\tilde{\phi}_{n} \cdot \tilde{\beta}_{n} \cdot \tilde{\gamma}_{n} \cdot \tilde{\tilde{\phi}}_{n}^{-1}$ is a continuous curve starting at $\tilde{x}_{n}$. Since $\tilde{\alpha}_{n}(0)=\tilde{\omega}_{n}(0)$ and the projections of $\tilde{\alpha}_{n}$ and $\tilde{\omega}_{n}$ to $M$ are homotopic, it follows that $\tilde{\alpha}_{n}(1)=\tilde{\omega}_{n}(1)$.

There are constants $1<\lambda<\mu$ such that

$$
\left\|T f^{n} v\right\|<\lambda^{n}<\mu^{n}<\left\|T f^{n} w\right\|
$$

for unit vectors $v \in E^{c}, w \in E^{u}$ and sufficiently large $n$. Then

$$
d\left(\tilde{\alpha}_{n}(0), \tilde{\alpha}_{n}(1)\right) \leq \text { length } \tilde{\alpha}_{n} \leq \lambda^{n} \text { length } \alpha
$$

and, if $Q$ is the constant of quasi-isometry for $W^{u}$,

$$
\begin{aligned}
d\left(\tilde{\omega}_{n}(0), \tilde{\omega}_{n}(1)\right) & \geq d\left(\tilde{\gamma}^{n}(0), \tilde{\gamma}^{n}(1)\right)-\text { length } \tilde{\beta}^{n}-\text { length } \tilde{\phi}^{n}-\operatorname{length} \tilde{\tilde{\phi}}^{n} \\
& \geq Q^{-1} \mu^{n} d_{u}(\gamma(0), \gamma(1))-\lambda^{n} \text { length } \beta-2 \operatorname{diam} M .
\end{aligned}
$$

For large $n$, these two estimates yield a contradiction. This finishes the case when $E^{s}=0$. The case where $E^{u}=0$ follows by analogy.

In the case that both $E^{u}$ and $E^{s}$ are non-zero, first observe that the foliations $W^{c s}$ tangent to $E^{s} \oplus E^{c}$ and $W^{c u}$ tangent to $E^{c} \oplus E^{u}$ are without holonomy, as each can be regarded as center foliation of a system which is partially hyperbolic in the weak sense. Then, $W^{c}$ is without holonomy as it is the intersection of two transverse foliations without holonomy.

We now prove Theorem 1.7. That is, assuming Global Product Structure and that $W^{c}$ is without holonomy, we show that all of the center leaves are homeomorphic.

Proof of Theorem 1.7. Let $p: \tilde{M} \rightarrow M$ be the universal covering map and fix $x_{0}, y_{0} \in \tilde{M}$. Our goal is to construct a homeomorphism $h: W^{c}\left(x_{0}\right) \rightarrow$ $W^{c}\left(y_{0}\right)$ such that for $x_{1}, x_{2} \in W^{c}(x), p\left(x_{1}\right)=p\left(x_{2}\right)$ if and only if $p\left(h\left(x_{1}\right)\right)=$ $p\left(h\left(x_{2}\right)\right)$. Then, $h$ will descend to a homeomorphism of the leaves $W^{c}\left(p\left(x_{0}\right)\right)$ and $W^{c}\left(p\left(y_{0}\right)\right)$ on $M$. 
First, consider the case where $x_{0}$ and $y_{0}$ lie on the same center-unstable leaf (which is the only case if $E^{s}=0$ ). Define $h$ as the map which assigns $x \in W^{c}\left(x_{0}\right)$ to the unique intersection of $W^{u}(x)$ and $W^{c}\left(y_{0}\right)$. This map exists and is continuous due to the Global Product Structure.

Suppose $x_{1}, x_{2} \in W^{c}\left(x_{0}\right)$ satisfy $p\left(x_{1}\right)=p\left(x_{2}\right)$. Take paths $\alpha:[0,1] \rightarrow$ $W^{c}(x)$ and $\beta:[0,1] \rightarrow W^{u}(x)$ such that $\alpha(0)=\beta(0)=x_{1}, \alpha(1)=x_{2}$ and $\beta(1)=h\left(x_{1}\right)$.

Define $\phi:[0,1] \times[0,1] \rightarrow \tilde{M}$ by $\phi(s, t)=W^{u}(\alpha(s)) \cap W^{c}(\beta(t))$. Again, Global Product Structure guarantees that this is well-defined and continuous. Consider the set

$$
S=\{t \in[0,1]: p(\phi(0, t))=p(\phi(1, t))\} .
$$

It is closed due to the continuity of $p$ and $\phi$. The assumption that the center foliation is without holonomy implies that $S$ is open in the relative topology of $[0,1]$, and as $0 \in S$ by the definition of $\phi$, it follows that $S=[0,1]$. In particular,

$$
p\left(h\left(x_{1}\right)\right)=p(\phi(0,1))=p(\phi(1,1))=p\left(h\left(x_{2}\right)\right) .
$$

By reversing the roles of $x_{0}$ and $y_{0}$, it is easy to find an inverse for $h$ and show that $p\left(h\left(x_{1}\right)\right)=p\left(h\left(x_{2}\right)\right)$ implies $p\left(x_{1}\right)=p\left(x_{2}\right)$. Thus, $h$ descends to a homeomorphism of $W^{c}\left(p\left(x_{0}\right)\right)$ and $W^{c}\left(p\left(y_{0}\right)\right)$ as center leaves on the manifold $M$.

That finishes the case of two center leaves on the same center-unstable leaf. The same argument applies to center leaves on the same center-stable leaf, and then, by composing such homeomorphisms, one can construct a homeomorphism between any two center leaves on $M$.

The last task of this section is to prove Theorem 1.10. We use the following classification given by Parwani [16, Theorem 1.10].

Theorem 4.2 (Parwani, [16]). Suppose $f: M \rightarrow M$ is partially hyperbolic with $\operatorname{dim} E^{s}=\operatorname{dim} E^{c}=\operatorname{dim} E^{u}=1$.

1. If $\pi_{1}(M)$ is solvable, $M$ is finitely covered by a torus bundle over the circle.

2. If $\pi_{1}(M)$ is nilpotent, $M$ is a circle bundle over the torus.

The first step in proving Theorem 1.10 is to classify the possible leaves of the foliations.

Proposition 4.3. Suppose $f: M \rightarrow M$ is partially hyperbolic with Global Product Structure and $\operatorname{dim} E^{s}=\operatorname{dim} E^{c}=\operatorname{dim} E^{u}=1$. Then, every leaf of $W^{c s}$ is either a plane, a cylinder, or a Möbius band.

Remark. This is a statement about leaves on $M$, not the universal cover $\tilde{M}$ where the leaves are all planes.

Remark. In the proof, we use the known fact that each leaf of an $n$-dimensional stable or unstable foliation is homeomorphic to $\mathbb{R}^{n}$. 
Proof. Fix $S \subset M$ a $c s$-leaf and $L$ a center leaf lying in $S$. The foliation $W^{c s}$ is Reebless [3]. Therefore, the embedding $S \hookrightarrow M$ induces an injection $\pi_{1}(S) \hookrightarrow \pi_{1}(M)$ [7][18. Let $\tilde{L} \subset \tilde{M}$ be a connected component of the preimage of $L$ under the universal covering map $p: \tilde{M} \rightarrow M$. Then $\pi_{1}$-injectivity implies $\tilde{L}$ is simply connected and therefore a line. For distinct points $x, y \in \tilde{L}$, the stable manifolds $W^{s}(x)$ and $W^{s}(y)$ are disjoint subsets of $\tilde{M}$ and therefore $\tilde{S}:=\bigcap_{x \in \tilde{L}} W^{s}(x)$, a line bundle over a line, is topologically a plane. It follows from Global Product Structure that $\tilde{S}$ is a connected component of $p^{-1}(S)$ and that $p(\tilde{S})=S$.

The set $\tilde{S}$ can be viewed as the universal cover of $S$ and each $\alpha \in \pi_{1}(S)$ can be viewed as a deck transformation $\alpha: \tilde{S} \rightarrow \tilde{S}$. Define a group action of $\pi_{1}(S)$ on $\tilde{L}$ by defining $\alpha \cdot x$ as the unique intersection of $W^{s}(\alpha(x))$ and $L$. If $\alpha \cdot x=x$, then $\alpha(x)$ and $x$ would lie on the same stable leaf and, assuming $\alpha$ is non-trivial, projection down to $M$ would yield a stable leaf which is a closed circle, a contradiction. Therefore, the action of $\pi_{1}(S)$ on the line $L$ is free. A classic theorem of Hölder concerning such actions implies that $\pi_{1}(S)$ is abelian (for instance, see [10, Theorem 6.10]).

If the subbundle $E^{u}$ is not orientable, there is a double cover of $M$ for which the lift of $E^{u}$ is orientable. Further, there is a unique lift of $f$ to a diffeomorphism of the cover which preserves this orientation; this map is also partially hyperbolic. Thus, modulo a finite cover, we may assume $E^{u}$ is orientable. By the same reasoning, assume $E^{c s}$ is orientable as well. Then the $c s$-leaf $S$ is orientable with an abelian fundamental group and is either a plane, a cylinder, or a torus.

Assume for the purpose of contradiction that $S$ is a torus. By Global Product Structure, every unstable leaf in $\tilde{M}$ intersects $\tilde{S}$ and therefore every unstable leaf in $M$ intersects $S$. The orientation of $E^{u}$ defines a flow along unstable leaves, and $S$ can be viewed as a global cross section. This defines a first return map $\phi: S \rightarrow S$. If the induced map $\phi_{*}: H_{1}(S) \rightarrow H_{1}(S)$ on the first homology group is hyperbolic, the Lefschetz fixed point theorem implies that the flow along unstable leaves has a periodic orbit, but this is impossible. If $\phi_{*}$ is not hyperbolic, one can show that $\pi_{1}(M)$ is nilpotent. Then by Theorem $4.2, M$ is a nilmanifold, and the classification results in [12] and 11] imply that no $c s$-leaf on such a manifold can be a torus.

We have shown that, when lifted to a finite cover, every leaf of $W^{c s}$ is either a plane or a cylinder, from which the result follows.

Proof of Theorem 1.10, It is a property of codimension-one foliations without holonomy that if $S$ is a leaf, then $\pi_{1}(S) \hookrightarrow \pi_{1}(M)$ is injective and, after identifying $\pi_{1}(S)$ with its image, that $\left[\pi_{1}(M), \pi_{1}(M)\right] \subset \pi_{1}(S)$. See [14 for a proof in the $C^{0}$ setting.

By Proposition 4.3, $\pi_{1}(S)$ is cyclic for any leaf $S$ of $W^{c s}$. Therefore, the commutator subgroup $\left[\pi_{1}(M), \pi_{1}(M)\right]$ is cyclic, and the fundamental group is solvable. Theorem 4.2 implies, in particular, that $\pi_{1}(M)$ is infinite. A classification of all possible solvable fundamental groups arising from 3-manifolds is 
given in [8]. If such a group is infinite and has cyclic commutator subgroup, it is nilpotent. Then, Theorem 4.2 completes the proof.

\section{Examples}

The Heisenberg group $\mathcal{H}$ consisting of matrices of the form

$$
\left(\begin{array}{lll}
1 & x & z \\
0 & 1 & y \\
0 & 0 & 1
\end{array}\right)
$$

is a Lie group. The corresponding Lie algebra $\mathfrak{h}$ is generated by the elements

$$
X=\left(\begin{array}{lll}
0 & 1 & 0 \\
0 & 0 & 0 \\
0 & 0 & 0
\end{array}\right), \quad Y=\left(\begin{array}{ccc}
0 & 0 & 0 \\
0 & 0 & 1 \\
0 & 0 & 0
\end{array}\right), \quad \text { and } \quad Z=\left(\begin{array}{ccc}
0 & 0 & 1 \\
0 & 0 & 0 \\
0 & 0 & 0
\end{array}\right)
$$

with $[X, Y]=Z$ and all other brackets zero. The Lie algebra can also be viewed as the set of those vector fields which are invariant under the derivative $d L_{g}$ : $T \mathcal{H} \rightarrow T \mathcal{H}$ of the action of left multiplication of the group $L_{g}: \mathcal{H} \rightarrow \mathcal{H}, h \mapsto g h$. As vector fields, $X, Y$, and $Z$ integrate to one-dimensional foliations on $\mathcal{H}$.

Define a Riemannian metric on $\mathcal{H}$ by declaring $X, Y$, and $Z$ to form an orthonormal basis at each point $p \in \mathcal{H}$. One can verify that the foliations tangent to $X$ and $Y$ are quasi-isometric, but the foliation tangent to $Z$ is not. Viewing a two-dimension subspace of $\mathfrak{h}$ as a subbundle of $T \mathcal{H}, X \oplus Z$ and $Y \oplus Z$ are each integrable and the resulting two-dimensional foliations are not quasi-isometric.

In [17, S. Smale describes a six-dimensional nilmanifold supporting Anosov diffeomorphisms. It is covered by the Lie group $\mathcal{H} \times \mathcal{H}$ consisting of two copies of the Heisenberg group. The corresponding Lie algebra $\mathfrak{h} \times \mathfrak{h}$ is generated by the elements $X_{1}, Y_{1}, Z_{1}, X_{2}, Y_{2}, Z_{2}$ where $\left[X_{1}, Y_{1}\right]=Z_{1},\left[X_{2}, Y_{2}\right]=Z_{2}$, and all other brackets are zero. There is a lattice $\Gamma \subset \mathcal{H} \times \mathcal{H}$ defining a compact nilmanifold $N:=(\mathcal{H} \times \mathcal{H}) / \Gamma$ and a constant $\lambda>1$ associated to $\Gamma$ such that for any $a, b \in \mathbb{Z} \backslash\{0\}$, the Lie algebra automorphism

$$
\begin{aligned}
X_{1} & \mapsto \lambda^{a} X_{1} & X_{2} & \mapsto \lambda^{-a} X_{2} \\
Y_{1} & \mapsto \lambda^{b} Y_{1} & Y_{2} & \mapsto \lambda^{-b} Y_{2} \\
Z_{1} & \mapsto \lambda^{a+b} Z_{1} & Z_{2} & \mapsto \lambda^{-a-b} Z_{2}
\end{aligned}
$$

induces a Lie group automorphism which takes $\Gamma$ to itself. Therefore, each $a, b \in \mathbb{Z} \backslash\{0\}$ defines a diffeomorphism $f_{a, b}: N \rightarrow N$ on the nilmanifold. If $a+b \neq 0$, one can verify that $f_{a, b}$ is Anosov.

In particular, if $a=1$ and $b=2$, then $f_{a, b}$ is Anosov with the splitting

$$
E^{u}=\left\langle X_{1}, Y_{1}, Z_{1}\right\rangle \text { and } E^{s}=\left\langle X_{2}, Y_{2}, Z_{2}\right\rangle
$$


and the resulting stable and unstable foliations are quasi-isometric. If, instead, $a=2$ and $b=-5$, then the splitting is

$$
E^{u}=\left\langle X_{1}, Y_{2}, Z_{2}\right\rangle \text { and } E^{s}=\left\langle X_{2}, Y_{1}, Z_{1}\right\rangle
$$

and neither foliation is quasi-isometric.

In either of these two examples, the diffeomorphism can alternatively be viewed as partially hyperbolic by grouping $X_{1}$ and $X_{2}$ into a center bundle. Thus, a dynamically coherent partially hyperbolic system on a nilmanifold may or may not have quasi-isometric stable and unstable foliations.

Acknowledgements The author would like to thank Enrique Pujals, Ali Tahzibi, and Amie Wilkinson for helpful discussions, as well as the anonymous reviewer for suggestions to improve the paper's presentation. The author gratefully acknowledges the financial support of CNPq (Brazil).

IMPA, Estrada Dona Castorina 110, Rio de Janeiro / Brasil 22460-320

\section{References}

[1] M. Brin. Nonwandering points of Anosov diffeomorphisms. Astérisque, 49:11-18, 1977.

[2] M. Brin. On dynamical coherence. Ergod. Th. and Dynam. Sys., 23:395401, 2003.

[3] M. Brin, D. Burago, and S. Ivanov. On partially hyperbolic diffeomorphisms of 3-manifolds with commutative fundamental group. Modern dynamical systems and applications, pages 307-312, 2004.

[4] M. Brin, D. Burago, and S. Ivanov. Dynamical coherence of partially hyperbolic diffeomorphisms of the 3-torus. Journal of Modern Dynamics, 3(1):1-11, 2009.

[5] M. Brin and Ja. Pesin. Partially hyperbolic dynamical systems. Math. USSR Izvestija, 8:177-218, 1974.

[6] D. Burago and S. Ivanov. Partially hyperbolic diffeomorphisms of 3manifolds with abelian fundamental groups. Journal of Modern Dynamics, 2(4):541-580, 2008.

[7] A. Candel and L. Conlon. Foliations II. American Mathematical Society, 2003.

[8] B. Evans and L. Moser. Solvable fundamental groups of compact 3manifolds. Transactions of the American Mathematical Society, 168:189210,1972 .

[9] J. Franks. Anosov diffeomorphisms. Global Analysis: Proceedings of the Symposia in Pure Mathematics, 14:61-93, 1970. 
[10] É. Ghys. Groups acting on the circle. Enseign. Math.(2), 47(3-4):329-407, 2001.

[11] A. Hammerlindl. Partial hyperbolicity on 3-dimensional nilmanifolds. arXiv:1103.3724.

[12] A. Hammerlindl. Leaf conjugacies on the torus. PhD thesis, University of Toronto, 2009.

[13] M. Hirsch, C. Pugh, and M. Shub. Invariant Manifolds, volume 583 of Lecture Notes in Mathematics. Springer-Verlag, 1977.

[14] T. Li. Commutator subgroups and foliations without holonomy. Proc. Amer. Math. Soc., 130(8):2471-2478, 2002.

[15] A. Manning. There are no new Anosov diffeomorphisms on tori. Amer. J. Math., 96(3):422-42, 1974.

[16] K. Parwani. On 3-manifolds that support partially hyperbolic diffeomorphisms. Nonlinearity, 23:589-606, 2010.

[17] S. Smale. Differentiable dynamical systems. Bull. Amer. Math. Soc., $73: 747,817,1967$.

[18] VV Solodov. Components of topological foliations. Mathematics of the USSR-Sbornik, 47:329, 1984. 